\title{
EDITORIAL
}

\section{Probiotics and obesity: a link?}

\author{
Didier Raoult cautions that the use of probiotics as growth promoters in the farming \\ industry means that further studies should be carried out before they are regarded as safe \\ for use in humans.
}

Recent studies on the human gut microbiota have shown that obesity is associated with a reduction in Gram-negative bacteria, specifically members of the Bacteroidetes, and an increase in Gram-positive Firmicutes ${ }^{1}$. Additionally, it has been shown that the gut microbiota of obese individuals is less diverse than that of non-obese individuals ${ }^{2}$. The manipulation of the gut microbiota - through the administration of probiotics and antibiotics - has been used for growth promotion in farm animals for 50 years and is regulated by the Food and Drug Administration (FDA) in the United States and by the European Commission in Europe. The probiotics used for this purpose in the farming industry include products containing Firmicutes, in particular Lactobacillus spp., Bifidobacterium spp. and Enterococcus spp. These products have been marketed and used in most of the animal farming industry, including in the production of poultry, calves and pigs, and many studies have shown increases in the size and weight of the young animals that are given these bacterial additives. Antibiotics have also been used for this purpose, although this practice is now banned in Europe.

Firmicutes are also used directly as therapeutic adjuvants in humans, under the names probiotics, prebiotics or, more generally, 'functional foods'. In the United States, these products are categorized by the FDA as 'generally regarded as safe' (GRAS; ironically, 'gras' translates as 'fat' in French). Analysis of these products showed that they contain high concentrations of live Lactobacillus spp. and Bifidobacterium spp. (up to $10^{8}$ organisms per gram or millilitre). These concentrations are similar to those used in animals as growth promoters. In the United States, probiotic-containing products such as dairy drinks or yogurts typically contain $>10^{7}$ lactobacilli. Lactobacillus acidophilus is found in functional foods in amounts that are equivalent to those used to cause weight gain in piglets. Lactobacillus spp. have also been associated with weight gain in children treated for diarrhoea ${ }^{3}$. In addition, some studies have demonstrated weight increases in children who received Lactobacillus rhamnosus, independent of the disease for which this probiotic was prescribed ${ }^{4}$. When these data are considered in the context of the epidemic of childhood obesity that is occurring in many developed countries, it seems essential to quickly and more completely study the effects of probiotics in the paediatric population.
Functional foods, including fermented dairy products containing probiotics, are gaining popularity in many countries, among children in particular, but little research has been carried out on the connection between these products and weight gain. These food products are often sold under the guise of having positive effects on children's health, but there are little conclusive data to support these claims. Surprisingly, the level of regulation for the use of probiotics in humans is less strict than that for their use in animals. The specific bacterial species involved and the concentrations at which they are present are often not made clear to consumers, and to my knowledge the long-term effects of probiotics as human food supplements or as adjunctive therapy have never been rigorously evaluated. In my opinion, further work using experimental models should be carried out to evaluate the role of these products as animal growth promoters before they are recommended for use in children.

It is my view that there is a danger that we may be causing a real human health problem by promoting for human consumption products containing bacteria that have been associated with weight gain in the animal food industry. Any chemical compound with such a side effect in experimental animals would be rigorously tested before being allowed to be used in food. I think that before probiotic and prebiotic products can be regarded as safe, it is imperative that they are tested in experimental models that evaluate the propensity of these products to cause obesity in humans.

\footnotetext{
1. Ley, R. E. et al. Human gut microbes associated with obesity. Nature 444, 1022-1023 (2006).

2. Turnbaugh, P. J. et al. A core gut microbiome in obese and lean twins. Nature 457, 480-484 (2009).

3. Chouraqui, J. P. et al. Assessment of the safety, tolerance, and protective effect against diarrhea of infant formulas containing mixtures of probiotics or probiotics and prebiotics in a randomized controlled trial. Am. J. Clin. Nutr. 87, 1365-1373 (2008).

4. Guandalini, S. et al. Lactobacillus GG administered in oral rehydration solution to children with acute diarrhea: a multicenter European trial. J. Pediatr. Gastroenterol. Nutr. 30, 54-60 (2000).
}

Didier Raoult is at Unité de Recherche en Maladies Infectieuses et Tropicales Émergentes, CNRS-IRD UMR 6236, Faculté de Médecine, Université de la Méditerranée, 27 Boulevard Jean Moulin, 13385 Marseille, France.

e-mail: didier.raoult@gmail.com as animal growth

before they are recommended for use in children. 